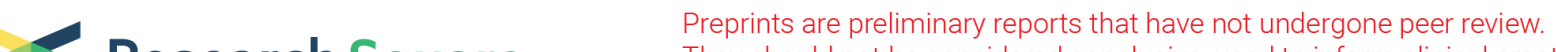 $\begin{array}{ll}\text { Research Square } & \text { They should not be considered conclusive, used to inform clinical practice, } \\ \text { or referenced by the media as validated information. }\end{array}$
}

\section{Neurogenesis and Proliferation of Neural Stem/Progenitor Cells Conferred by Artesunate via FOX03a/p27Kip1 Axis in Mouse Stroke Model}

\section{Kaiyuan Zhang}

Southwest hospital, third militery medical university

\section{Yang Yang}

Third Military Medical University Southwest Hospital

Hongfei Ge

Southwest hospital, Third military medical university

Ju Wang

Third Military Medical University Southwest Hospital

\section{Xuejiao Lei}

Third Military Medical University Southwest Hospital

\section{Xuezhu Chen}

Third Military Medical University Southwest Hospital

\section{Feng Wan}

University of Macau Faculty of Science and Technology

\section{Hua Feng}

Third Military Medical University Southwest Hospital

Liang Tan ( $\square$ tracy200712@hotmail.com )

Third Military Medical University Southwest Hospital https://orcid.org/0000-0002-6916-2134

\section{Research Article}

Keywords: Artesunate, neural stem/progenitor cells, white matter injury, stroke, FOXO3a/p27kip1 axis

Posted Date: November 12th, 2021

DOI: https://doi.org/10.21203/rs.3.rs-1022332/v1

License: (c) (1) This work is licensed under a Creative Commons Attribution 4.0 International License. Read Full License 


\section{Abstract}

Promoting neurogenesis and proliferation of endogenous neural stem/progenitor cells (NSPCs) is considered a promising strategy for neurorehabilitation after stroke. Our previous study revealed that a moderate dose of artesunate (ART, $150 \mathrm{mg} / \mathrm{kg}$ ) could enhance functional recovery in middle cerebral artery occlusion (MCAO) mice. This study aimed to investigate the effects of ART treatment on neurogenesis and proliferation of NSPCs using a rodent MCAO model. MRI results indicated that the ischemic brain volume of MCAO mice was reduced by ART treatment. The results of diffusion tensor imaging, electron microscopic, and immunofluorescence of Tuj-1 also revealed that ischemia-induced white matter lesion was alleviated by ART treatment. After ischemia/reperfusion, endogenous NSPCs were activated by ART, which was displayed by comparing the proportion of Brdu+ neuronal precursor cells in the ipsilateral subventricular zone and peri-infarct cortex. Furthermore, the neuro-restorative effects of ART were abolished by the overexpression of FOXO3a. These findings suggested that ART could rescue penumbra damage and alleviate white matter injury, subsequently contributing to poststroke functional recovery by promoting neurogenesis and proliferation of endogenous NSPCs via the FOX03a/p27 Kip1 pathway.

\section{Introduction}

As recently reported by the American Heart Association (AHA), the mean global lifetime risk of stroke has increased by $8.9 \%$ from 1999 to 2016 [1]. Post-stroke neurologic deficit causes a massive social and family burden, while neuro-functional recovery has been intensively investigated [2]. Neural stem/progenitor cells (NSPCs) can be self-renewal and differentiate into mature neurons, oligodendrocytes, and astrocytes [3]. Previous studies have indicated that NSPCs could be a novel candidate of cell therapy approaches for hypoxic-ischemic brain injuries [4]. However, the neurogenesis and differentiation of endogenous NSPCs are remarkably disrupted in an impaired neurovascular microenvironment after hypoxic-ischemic brain injuries [5].

Artesunate (ART), a derivative of artemisinin, exhibits high efficacy and low toxicity in malarial treatment [6]. ART was approved for first-line medical use of malaria by the US Food and Drug Administration in 2020 for its low mortality and high toleration than quinine in several trials $[7,8]$. The ability to penetrate the blood-brain barrier is commonly used in patients with cerebral malaria [9]. ART's potential tumorsuppressive roles have been widely studied [10], as well as its effects on organ injury [11], immune responses [12], and neurotransmission regulation [13]. Our previous study has suggested that ART restores the damaged blood-brain barrier by regulating the S1pR/PI3K signaling pathway in a mouse model of subarachnoid hemorrhage [14]. Moreover, our recent findings have revealed that the proliferation of NSPCs and neurogenesis are promoted by ART-modulated PI3K/Akt/FOXO-3a/p27kip1 signaling in an in vitro oxygen/glucose deprivation model [15]. However, ART treatment's neuroprotective roles on endogenous NSPCs in the subventricular zone (SVZ) and white matter injury have not been investigated using an animal stroke model. 
FOXO transcriptional factors are the targets of the PI3K/Akt signaling pathway [16]. Upon dephosphorylation, FOXO3a is translocated into the nucleus to induce the expression of the cyclindependent kinase inhibitor 1B (p27kip1) [17]. Subsequently, the formation of a cyclinE-CDK2 complex induced by $\mathrm{p} 27 \mathrm{kip} 1$ could trigger cell cycle arrest at G0/G1 phase, suppressing neurogenesis and proliferation of NSPCs [18]. Inhibition of FOXO3a/p2 $27^{\text {Kip } 1}$ signaling could break the cell cycle arrest in endogenous neural stem/progenitor cells induced by ischemia/reperfusion.

Based on these findings, we hypothesized that ART could rescue the penumbra damage, alleviate white matter injury, and reverse neurologic impairment after ischemic stroke. The present study aimed to investigate ART's potential neuroprotective functions and the underlying mechanisms using an MCAO mouse model.

\section{Materials And Methods}

\section{Establishment of animal model}

A total of 348 C57BL/6J male mice (12 to 16-week-old; 25-28g; Jackson Laboratory, stock no. 000664) were purchased from the Animal Center of the Third Military Medical University. Mice were housed in a pathogen-free environment and had free access to food and water with the light/dark cycle of 12:12 hours.

As previously described, the MCAO model was used to generate focal unilateral cerebral ischemia [19]. Briefly, C57BL/ 6 mice were anesthetized using a $2 \%$ isoflurane/air mixture (1-2 L/min). A vertical midline incision exposed the submandibular glands on the neck. The carotid sheath was removed, and the common carotid artery was separated to avoid accidental injury to the vagus nerve. A 2.0-cm siliconecoated nylon suture with a diameter of $0.23 \mathrm{~mm}$ (Sunbio Co., Ltd., Beijing, China) was gently inserted from the external carotid artery stump internal carotid artery, which terminated at the beginning of the middle cerebral artery. The cerebral blood flow was blocked for 60 minutes. The same procedures were performed on sham group mice apart from occlusion. The temperature of experimental animals was monitored during the surgery and the recovery from anesthesia. All surgeries and tests were carried out randomly by blinded researchers.

\section{Experimental grouping and design}

\section{Experiment 1}

To evaluate the recovery of motor function in MCAO animals treated with different ART concentrations, mice were randomly sorted into five groups: Sham $(n=10), M C A O+V e h$ (saline, $n=10) ; M C A O+50 \mathrm{mg} / \mathrm{kg}$ ART $(n=10) ; M C A O+150 \mathrm{mg} / \mathrm{kg}$ ART $(n=10)$ and MCAO+250mg/kg ART $(n=10)$. The ART (HolleyWulingshan pharmaceuticals Ltd., Chongqing, China) was given intraperitoneally once per day, and the first dose was given immediately after reperfusion. Furthermore, each group's recovery of forelimb motor 
function was assessed using cylinder task, grid-walking task pasta handing, and single-pellet retrieval task. Animals were tested once on these tasks 1 week before surgery to establish baseline performance levels and were then tested at 3 and 7 days post-MCAO. Behaviors were scored by observers blinded to the grouping (Fig S1A of Supplemental Material). Detailed descriptions were presented in supplementary materials.

\section{Experiment 2}

To further examine the efficacy of ART and investigate the underlying mechanisms of its neuroprotective roles, the established MCAO model mice were randomly grouped: Sham, MCAO+Veh (saline; Ad-Flag,), MCAO+ART (150mg/kg; Ad-Flag) and MCAO+ART +FOXO3aOE (150mg/kg/day; Ad-TM-FOXO3a). T2weight MRI was used to measure the cerebral infarcted volume. Diffusion tensor imaging (DTI), transmission electron microscopy, and immunofluorescence assay were performed to examine white matter injury. Immunofluorescence staining and western blotting (WB) were carried out to assess the proliferation of NSPCs in the SVZ and the perilesional cortex, which revealed the proliferation and migration of NSPCs after MCAO. FOXO3a/p27kip1-associated molecules' expression levels were determined at three days post-MCAO (Fig S1B of Supplemental Material).

To further investigate ART's effects on the stroke model, the three experimental groups: sham, $\mathrm{MCAO}+\mathrm{Veh}$, and $\mathrm{MCAO}+\mathrm{ART}$, were assessed by comparing by neuro-function, infracted volume, and white matter injury. Additionally, MCAO+ART and $\mathrm{MCAO}+\mathrm{ART}+\mathrm{FOXO} \mathrm{a}^{\mathrm{OE}}$ groups were also examined to elucidate the underlying mechanisms of ART-induced neuroprotective roles.

\section{Magnetic resonance imaging (MRI) and diffusion tensor imaging (DTIX}

Mice were anesthetized using a $2 \%$ isoflurane/air mixture before MRI. Serial and T2-weighed imaging was carried out on 16 coronal slices (thickness $=1.0 \mathrm{~mm}$ ) using a 7.0-T Varian MR scanner (field of view $=$ $35 \times 25 \mathrm{~mm}$; Bruker Analytik $\mathrm{GmbH}$, Karlsruhe, Germany) at 72h after MCAO.

DTI assay enabled the visualization and characterization of white matter fibers in three dimensions and was performed as previously described [20]. Diffusion Toolkit software (Harvard Medical School, Boston, US) was used to track white matter fiber bundles and calculate apparent diffusion coefficient (ADC) and the fractional anisotropy (FA) values in the ipsilateral internal capsule. The FA value (Ipsil/contral) was calculated as (FA value at ipsilateral IC)/ (FA value at contralateral internal capsule) x100\%.

\section{2, 3, 5-triphenyl tetrazolium hydrochloride (TTC) staining}

At 72h after MCAO, TTC staining was performed as previously described[21]. Brains were immediately removed following anesthetization, sectioned into coronal slices with $1 \mathrm{~mm}$ intervals. The slides were 
further incubated with $2 \%$ 2,3,5-triphenyl tetrazolium hydrochloride (Sigma-Aldrich, St. Louis, MO) at $37^{\circ} \mathrm{C}$ for $30 \mathrm{~min}$ in the dark.

\section{Transmission electron microscopy}

At $72 \mathrm{~h}$ after MCAO, mice were anesthetized and perfused with pre-cooled $0.01 \mathrm{M}$ PBS and $4 \%$ formaldehyde. After the perfusion, brain tissues were obtained and immersed in glutaraldehyde. The perinfracted cerebral cortex was immediately removed from the solution. Subsequently, the white matter around the cerebral infarction was homogenized into small pieces $\left(\sim 1 \mathrm{~mm}^{3}\right)$ and then stored in $2 \%$ formaldehyde and $2 \%$ at glutaraldehyde at $4^{\circ} \mathrm{C}$ overnight. After dehydration, samples were impregnated with epoxy resin, sectioned, and then double-stained using lead citrate and uranyl acetate. Images were captured with an $\mathrm{H}-7100$ transmission electron microscope (Hitachi, Tokyo, Japan) as previous described [22].

\section{Bromodeoxyuridine (BrdU) injection and incorporation assay}

$100 \mathrm{mg} / \mathrm{kg}$ of BrdU was administered intraperitoneally every $24 \mathrm{~h}$, and the first dose was given immediately after MCAO. The coronal sections (thickness $=25 \mu \mathrm{m}$ ) were stained. Staining of BrdU and DCX double-positive cells in the peri-infarct and SVZ regions was visualized on the confocal images. Cell counting was performed on four slices for each brain to calculate the relative percentage of stained cells in the two areas.

\section{Immunofluorescence assay}

Treated specimens of the peri-infarct brain region and SVZ were incubated with corresponding antibodies against BrdU (1:500, Millipore, Germany), DCX (1:500, Abcam, UK), Tuj-1 (1:1000, Abcam, UK) and Nestin (1:500, Abcam, UK) at $4^{\circ} \mathrm{C}$ overnight. The images were captured using a confocal microscope (Carl Zeiss, LSM780, Weimar, Germany)

\section{Western blotting}

Protein samples were extracted from the peri-infarct cortex of MCAO mice, separated on a $10 \%$ Bis-tris selective denaturing gel, and then transferred onto a PVDF membrane (Millipore Inc., MA, US). Bovine serum albumin (3\%) in TBST was used to block the membranes incubated at room temperature for 2 hours. The primary antibodies were then incubated: rabbit polyclonal antibody against Foxo-3a (R\&D, 1:1000), mouse monoclonal antibody against $p 27^{\mathrm{kip} 1}$ (CST, 1:2000), rabbit polyclonal antibody against Cyclin E (CST, 1:1000), rabbit polyclonal antibody against CDK2 (CST, 1:1000), rabbit polyclonal antibody against Nestin (Abcam, 1:2000) or mouse monoclonal antibody against GAPDH (Zsgb-bio, 1:1000) at $4^{\circ} \mathrm{C}$ overnight. The following day, the membrane was rinsed three times with TBST to remove excessive 
primary antibodies. Subsequently, the membrane was further incubated with HRP-labeled secondary antibody (Zsgb-bio, 1:5000) at room temperature for 2 hours. The signals were detected using an ECL reagent (Thermo Scientific, US) and visualized on the ChemiDoc ${ }^{T M} X R S+$ imaging system. Protein bands were quantified by densitometry using Quantity One software (Bio-Rad Laboratories, Inc., Hercules, CA, US).

\section{Adenovirus transfection}

An adenoviral vector expressing constitutively active FOXO3a(AD-TM-FOXO3a) was adopted; thus, TMFOXO3a could not be phosphorylated by p-Akt. Adenovirus Ad-TM (triple mutant)-FOXO3a (FOXO3a ${ }^{\mathrm{OE}}$ ) and negative control Ad-Flag were purchased from Shanghai Heyuan Biological Co., Ltd. In the FOXO3a overexpression vector, three conserved Akt phosphorylation sites (Thr-32, Ser-253, and Ser-315) of FOXO3a were replaced by alanine residues to prevent phosphorylating by p-Akt. Ad-TM-FOXO3a and AdFlag were injected into the lateral ventricles $(0.33 \mu \mathrm{l} / \mathrm{min})$ using a specific coordinate $(0.2 \mathrm{~mm}$ posterior to the bregma, $2 \mathrm{~mm}$ ventral) the skull, and $1 \mathrm{~mm}$ lateral to the sagittal line) with stereotaxic frame 48 hours before MCAO surgery.

\section{Statistical analysis}

All data were presented as the mean \pm standard error of the mean (SEM) and passed a normality test. Comparisons between two groups were analyzed using 2-tailed Student t-tests. Behavioral data collected at repeating time points were analyzed using two-way repeated-measures ANOVA, followed by the Bonferroni post hoc test. Other data were analyzed using one-way ANOVA followed by Tukey's post hoc test. The statistical analyses were conducted using SPSS v19.0 (SPSS Inc., Chicago, IL). P<0.05 was considered statistically significant.

\section{Results}

ART alleviated the motor dysfunction, reduced cerebral infarct volume, and white matter lesion after MCAO.

The results of cylinder task, grid-walking task, and pasta handing, single-pellet retrieval task (Fig S3 of Supplemental Material) indicated that only $150 \mathrm{mg} / \mathrm{kg}$ ART treatment remarkably promoted functional recovery at day 3 and 7 after ischemia/reperfusion. Those findings indicated that $150 \mathrm{mg} / \mathrm{kg}$ ART could improve the final fine motor function outcome in the sub-acute phase of stroke. Moreover, animals' survival rate and body weight in the MCAO+ART group were not reduced compared to the MCAO+Veh group ( $p>0.05$, Fig S2 of Supplemental Material).

The T2-weighted images revealed that the ischemic size of MCAO mice was reduced by ART treatment three days post-reperfusion ( $p<0.05$, Fig. $1 A$ and $B$ ). The ADC and FA values were calculated using three diagonalized diffusion tensor elements in the ipsilateral internal capsule (IC). Significant reduction and 
fragmentation of nerve fiber bundles were observed in the ipsilateral hemisphere three days following MCAO (Fig. 1C). Compared with the sham group, ADC value was remarkedly elevated, and FA value was notably decreased in infarcted IC at day $3(p<0.05)$, suggesting nerve fiber damage in ipsilateral IC. Moreover, $150 \mathrm{mg} / \mathrm{kg}$ of ART prevented white matter lesions induced by MCAO, as the reduction of ADC and FA values was reversed in the MCAO+ART group compared to the MCAO+Veh group $(p<0.05$; Fig. 2CE).

The axon and myelin sheath injury at the infract IC at day 3 after MCAO were analyzed by Electron microscopy, which indicated that treatment with $150 \mathrm{mg} / \mathrm{kg}$ ART alleviated axon swelling and myelin injury in infracted white matter fiber (Fig. 1F). Besides, the mean axonal number was significantly increased by ART treatment ( $<<0.05$; Fig. $1 \mathrm{G}$ ), whereas mean G-ratio (ratio of axon diameter to total outer diameter) $(\mathrm{p}<0.05$; Fig. $1 \mathrm{H})$ and axon diameter $(\mathrm{p}<0.05$; Fig. $1 \mathrm{I})$ were reduced compared with MCAO+Veh group. Furthermore, immunofluorescence staining of Tuj-1 revealed many axons and fractured dendrites, as well as an infarcted internal capsule at day 3 post-MCAO $(p<0.05$; Fig. $1 \mathrm{~J})$. The number of DAPI+ cells and the fluorescence intensity of Tuj-1 were significantly increased in the MCAO+ART group compared to the control $(p<0.05$; Fig. $1 \mathrm{~K}$ and $\mathrm{L})$.

\section{ART promoted post-MCAO neurogenesis through F0X03a/p27 Kip1 pathway.}

Normalized DCX intensity was significantly increased in ipsilateral SVZ by ART treatment $(p<0.05 ;$ Fig. $2 A$ and $\mathrm{B}$ ). Furthermore, the numbers of $\mathrm{DCX}^{+}$and $\mathrm{Brdu}^{+}$cells were measured to evaluate neuroblasts' proliferation after MCAO in the ipsilateral SVZ (Fig. 2A and C). The result indicated that $150 \mathrm{mg} / \mathrm{kg}$ of ART significantly promoted neurogenesis in ipsilateral SVZ after MCAO $(p<0.05)$. Furthermore, the BrdU incorporation assay was carried out to evaluate the proliferation of $\mathrm{DCX}^{+}$cells in the peri-lesioned cortex. Compared with the MCAO+Veh group, ART treatment notably increased the number of $\mathrm{DCX}^{+}$and $\mathrm{Brdu}^{+}$ cells and elevated DCX intensity in the perilesional cortex on day 3 after MCAO ( $<<0.05$; Fig. 2D-F). Furthermore, Nestin's expression levels, a specific marker for NSPCs, were significantly increased by ART treatment in the peri-infarct cortex $(p<0.05 ;$ Fig. $3 \mathrm{~A}$ and $\mathrm{F})$.

The result of WB in the peri-infarct cortex suggested that ART treatment decreased the expression of FOX03a $(p<0.05)$, which was reversed by the treatment with Ad-TM-FOXO3a (FOXO3aOE; $p<0.05$, Fig. 3A and B). Additionally, the expression levels of $p 27^{\mathrm{Kip} 1}$ were investigated, and the results indicated that ART treatment significantly reduced the expression of $p 27^{\mathrm{Kip} 1}(\mathrm{p}<0.05)$, and these effects were abrogated by FOX03 $\mathrm{a}^{\mathrm{OE}}$ ( $p<0.05$, Fig. $3 \mathrm{C}$ ). Our WB data revealed that the expression levels of Cyclin $E$ and CDK2 were remarkably upregulated by ART treatment $(p<0.05)$, which was abolished by $\mathrm{FOXO3}^{\mathrm{OEE}}(p<0.05$, Fig. 3D and E). Moreover, Nestin's ART-induced expression in the peri-infarct cortex was also reversed by the treatment with FOXO3a ${ }^{\mathrm{OE}}(\mathrm{p}<0.05$; Fig. $3 \mathrm{~F})$. Therefore, these WB data revealed that $A R T$ could promote post-MCAO neurogenesis in mice by regulating the FOXO3a/p27 kip1/Cyclin E/CDK2 pathway.

\section{The roles of FOXO3a/p27 Kip1 signaling on ART-induced neuroprotection in MCAO mice.}


The expression of FOXO3a in the mice's brain of the Ad-TM-FOXO3a group was significantly higher than the Ad-Flag group ( $p<0.05$; Fig S4 of Supplemental Material). The cerebral infarct volume was evaluated by T2-weighed imaging (Fig. 4A) and TTC staining (Fig. 4B) three days after MCAO, and overexpressed FOXO3a reversed ART-induced reduction of cerebral infarct volume (MCAO+ART+FOXO3a ${ }^{\mathrm{OE}}$ vs. MCAO+ART group, p<0.05; Fig. $4 \mathrm{C}$ and D). Exceptional motor function and sensitivity to injury of MCAO were also evaluated using the grid-walking and single-pellet retrieval tasks. The results indicated that overexpression of FOXO3a notably abolished the protective effects of ART treatment at 3 and 7 days post-MCAO ( $<<0.05$; Fig. 4E and $F)$.

Also, white matter lesions in the infarcted brain were examined by transmission electron microscopy (Fig. 5A). The results indicated that overexpressed FOXO3a significantly abolished the protective effects of ART on axon swelling and myelin injury in white matter fiber, and ART-induced impact on the mean

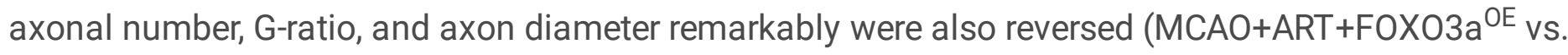
MCAO+ART group, $\mathrm{p}<0.05$; Fig. 5B-D). Post-MCAO neurogenesis was also evaluated by immunofluorescence staining of DCX and BrdU (Fig. 5E). The ART-induced increase of normalized DCX intensity and the numbers of $\mathrm{DCX}^{+}$and $\mathrm{Brdu}^{+}$cells in the peri-lesioned cortex were abolished by overexpressed FOXO3a at day 3 post-MCAO ( $p<0.05$; Fig. 5F). Moreover, increased BrdU intensity in ipsilateral SVZ caused by ART was also abolished by the overexpression of FOXO3a $(p<0.05$; Fig S5 of

\section{Supplemental Material).}

\section{Discussion}

Post-stroke functional recovery remains challenging, which poses a substantial threat to public health [23]. Therefore, it is essential to develop therapeutic strategies for patients with ischemic stroke. ART, a stable derivative of artemisinin with high efficacy and low toxicity, exerts anti-malarial functions and can penetrate the blood-brain barrier[24]. Apart from its anti-neuroinflammatory and antioxidant roles, ART can also protect the blood-brain barrier and promote neurogenesis; therefore, it is a novel candidate for treating neurological disorders, according to our previous report[25].

In Experiment I, three concentrations of ART $(50,150$, and $250 \mathrm{mg} / \mathrm{kg}$ ) were used to elucidate the effects of different doses on motor function recovery in MCAO mice. The recovery of each group's forelimb motor function was assessed using cylinder, grid-walking, and pasta-handling, single-pellet retrieval tasks. We found that ART at $150 \mathrm{mg} / \mathrm{kg}$ significantly promoted the delicate finger functional recovery at the subphase of stroke (Fig S3 of Supplemental Material). The cerebral infarcted volume and white matter injury were examined to assess ART's efficacy in the MCAO model. The results from T2-weight MRI indicated that the ischemic brain volume of MCAO mice was reduced by ART treatment three days after reperfusion (Fig. 1). The white matters, including the axon and myelin of cortical projection neurons, are vulnerable to ischemia/reperfusion lesion, which results in contralateral motor function deficiency after MCAO. Our results from DTI, electron microscopy and immunofluorescence staining of Tuj-1 suggested that $150 \mathrm{mg} / \mathrm{kg}$ of ART also alleviated post-MCAO white matter lesion in mice (Fig. 1). 
Furthermore, the proliferation and neurogenesis of NSPCs in the peri-infract region were significantly promoted by ART treatment at day 3 post-MCAO (Fig. 2). The bystander effects [26] of NSCPs may serve essential roles in reducing infarct volume in the early stroke stage, revealed by TTC staining and MRI (Fig. 4). The primary functions of NSCPs include protecting the blood-brain barrier[27] promoting the secretion of neurotrophic factors such as BDNF, NTFs, and VEGF [28], inhibiting local neuroinflammation [29] and enhancing the angiogenesis [30]. Therefore, activation of NSPCs by ART treatment could reduce the infarct volume at the early stage of stroke.

Moreover, previous studies have suggested that DCX is a microtubule-associated protein expressed by immature neurons or neuronal-restricted precursors that could not differentiate into glial cells [31]. In the present study, whether ART could promote post-MCAO neurogenesis was investigated. The numbers of DCX and BrdU double-stained cells in the ipsilateral SVZ and peri-lesioned cortex were compared three days after MCAO. The results indicated that ART could activate endogenous NSPCs to proliferate and differentiate rapidly in both the ipsilateral subventricular zone and perilesional cortex, subsequently reconstructing the neural network of the ischemic area (Fig. 2). The previous study has also revealed that dead neurons in the infarct cortex are replaced by regionally-induced NSPCs from cortical and subpial regions and leptomeninges (pia mater), rather than migration of NSPCs from the SVZ [32].

Furthermore, this review suggested that activation of NSPCs could induce neurogenesis in the periischemic areas but not in the ischemic core. Our data revealed that the proliferation and neurogenesis of endogenous NSPCs in SVZ were promoted by ART treatment and activated NSPCs relocated from SVZ to the peri-ischemic areas in MCAO mice (Fig. 2A). Furthermore, ART-induced neurogenesis in the periischemic regions was confirmed by the co-staining of DCX and Nestin (Fig. 2D and 5E). Although the proliferating immature neurons in the peri-infract area could be partially from SVZ-derived NSPCs, our data still suggested that ART could promote the proliferation and neurogenesis of SVZ-derived or in situ NSPCs after stroke.

FOXO3a, a key transcription factor, is phosphorylated by Akt. FOXO3a is dephosphorylated and translocated into the nucleus to regulate multiple gene transcription [33]. Cyclin-dependent kinase inhibitor 1B (p27 ${ }^{\mathrm{Kip} 1}$ ), which FOXO3a holds, belongs to the Cip/Kip family of cyclin-dependent kinase (CDK) inhibitor and binds to cyclin $E$, consequently downregulating cyclin $E$ and CDK2 at G1/G0 phase [34]. When NSPCs are stimulated by inflammation or hypoxia, FOXO3a is dephosphorylated and translocated into the nucleus to induce the $27^{\mathrm{Kip} 1}$ expression; thus, the cell cycle of NSPCs is arrested at G0/G1 phase [35]. To investigate the underlying mechanisms of ART-induced proliferation in NSPCs, the expression levels of FOXO3a/p27 $7^{\mathrm{kip} 1}$-associated molecules were determined using WB in MCAO mice. The activity of protein kinase complex CyclinE-CDK2 could be inhibited by cyclin-dependent kinase inhibitor 1B ( $\mathrm{p} 27^{\mathrm{Kip} 1}$ ), a KIP family member, and regulated by FOXO3a. Our results revealed that ART treatment caused inhibition of FOXO3a and downregulation of $\mathrm{p} 27^{\mathrm{Kip} 1}$, which subsequently promoted endogenous NSCPs proliferation in the SVZ and peri-infarct zone of MCAO mice (Fig. 2 and 3). 
To identify FOXO3a/p27Kip1 pathway involvement on ART-modulated neuroprotective roles in MCAO mice, T2-weight MRI, TTC assay, transmission electron microscopy, and immunofluorescence assay were performed to investigate whether overexpressed FOXO3a could reverse the neuroprotective effects of ART. Improved performance in behavior tests and reduced cerebral infarct volume caused by ART

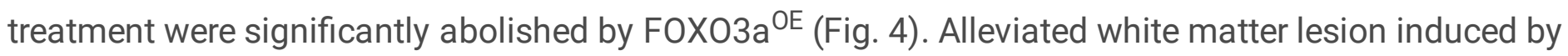
ART treatment was also abrogated by FOXO3a ${ }^{\mathrm{OE}}$ (Fig. 5A-D). Moreover, ART-induced normalization of $\mathrm{DCX}$ intensity was abolished by $\mathrm{FOXO} \mathrm{a}^{\mathrm{OE}}$ treatment (Fig. $5 \mathrm{E}$ and $\mathrm{F}$ ), suggesting that $\mathrm{ART}$ promoted post-

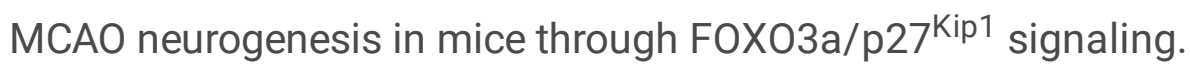

However, there were still some limitations in the present study. For example, ART treatment's effects and safety should be further investigated on the multiple animal stroke models, especially in nonhuman primates. Moreover, a comparison of the activating endogenous NSPCs and transplanting exogenous NSPCs should be performed in further study. We are focusing on the repair effects of the endogenous NSPCs neurogenesis and proliferation in the current study.

Neurogenesis and differentiation of endogenous NSPCs in the adult brain are considered a potential therapeutic approach for ischemic stroke, improving functional recovery and alleviating secondary brain injury. Upon activation, resting endogenous NSPCs in the SVZ niche could proliferate and migrate towards the lesion and further differentiate into neurons to participate in reconstructing infract white matter. However, the detailed functions of ART still require further investigation before clinical trials. The present study indicated that ART could rescue penumbra damage and alleviate white matter injury, promoting neurogenesis and contributing to post-stroke functional recovery through FOXO3a/p27 ${ }^{\mathrm{Kip} 1}$ pathway in MCAO mice (Fig. 6). These findings suggested that ART could be a novel therapeutic candidate for neurogenesis and functional reconstruction after stroke.

\section{Abbreviations}

NSPCs, neural stem/progenitor cells; ART, Artesunate; MCAO, middle cerebral artery occlusion; FOXO3a, forkhead box 03a; p27 kip1 , cyclin-dependent kinase inhibitor 1B; CDK2, cyclin-dependent Kinase 2; MRI, magnetic resonance imaging; DTI, diffusion tensor imaging; FA, fractional anisotropy values; ADC, apparent diffusion coefficient value; IC, internal capsule; SVZ, subventricular zone; SGZ, subgranular zone of hippocampal dentate gyrus; TTC, 2, 3, 5-triphenyltetrazolium hydrochloride; BrdU, bromodeoxyuridine; DCX, doublecortin; Tuj-1, neuron-specific class III beta-tubulin.

\section{Declarations}

\section{Funding}

This study was supported by the Military Medicine Pre-research Project of Army Medical University(2018XYY11), National Natural Science Foundation of China (81901267), and the Natural 
Science Foundation of Chongqing (Grant no. cstc2019jcyj-msxmX0458). The funders were not involved in study design, data collection/interpretation, and the decision to submit the publication results.

\section{Author contribution statement}

KYZ performed most of the experiments, with assistance from $Y Y$ and $X Z C X J L$ and $Y Y$ analyzed the results and produced the figures $\mathrm{KYZ}$ conducted statistical analysis FW, and HGF performed animal experiments XZC, XJC and HFG conducted immunoblotting and immunostaining KYZ drafted the manuscript $Y Y$ and $L T$ reviewed the manuscript $L T$, and HF designed experiments All authors approved the final version of the document

\section{Consent for publication}

All the authors declared no competing interests and agreed to publication.

\section{Consent to participate}

Not application

\section{Compliance with Ethical Standards}

The Ethics Committee of the Third Military Medical University approved all the animal work protocols (approval no. AMUWEC20201153). The experiments were performed in concordance with Animal Research standards: Reporting of In Vivo Experiments (ARRIVE) guidelines.

\section{Acknowledgements and Availability of data and materials}

Not applicable and the dataset used in this study were available from the corresponding author upon reasonable request.

\section{References}

1. Virani SS, Alonso A, Benjamin EJ, Bittencourt MS, Callaway CW, Carson AP, Chamberlain AM, Chang AR, Cheng S, Delling FN et al.: Heart Disease and Stroke Statistics-2020 Update: A Report From the American Heart Association. Circulation 2020, 141(9):e139-e596.

2. Tan L, Margaret B, Zhang JH, Hu R, Yin Y, Cao L, Feng H, Zhang Y: Efficacy and Safety of Cilostazol Therapy in Ischemic Stroke: A Meta-analysis. J Stroke Cerebrovasc Dis 2015, 24(5):930-938. 
3. Bond AM, Ming GL, Song H: Adult Mammalian Neural Stem Cells and Neurogenesis: Five Decades Later. Cell Stem Cell 2015, 17(4):385-395.

4. Zhu X, Yan J, Bregere C, Zelmer A, Goerne T, Kapfhammer JP, Guzman R, Wellmann S: RBM3 promotes neurogenesis in a niche-dependent manner via IMP2-IGF2 signaling pathway after hypoxicischemic brain injury. Nat Commun 2019, 10(1):3983.

5. Wang J, Hu J, Chen X, Lei X, Feng H, Wan F, Tan L: Traditional Chinese Medicine Monomers: Novel Strategy for Endogenous Neural Stem Cells Activation After Stroke. Front Cell Neurosci 2021, 15:628115.

6. Pekyi D, Ampromfi AA, Tinto H, Traoré-Coulibaly M, Tahita MC, Valéa I, Mwapasa V, Kalilani-Phiri L, Kalanda G, Madanitsa M et al: Four Artemisinin-Based Treatments in African Pregnant Women with Malaria. N Engl J Med 2016, 374(10):913-927.

7. Dondorp AM, Fanello Cl, Hendriksen IC, Gomes E, Seni A, Chhaganlal KD, Bojang K, Olaosebikan R, Anunobi $\mathrm{N}$, Maitland $\mathrm{K}$ et al: Artesunate versus quinine in the treatment of severe falciparum malaria in African children (AQUAMAT): an open-label, randomised trial. Lancet 2010, 376(9753):1647-1657.

8. Sinclair D, Donegan S, Isba R, Lalloo DG: Artesunate versus quinine for treating severe malaria. Cochrane Database Syst Rev 2012, 2012(6):Cd005967.

9. Drecourt A, Babdor J, Dussiot M, Petit F, Goudin N, Garfa-Traoré M, Habarou F, Bole-Feysot C, Nitschké P, Ottolenghi C et al: Impaired Transferrin Receptor Palmitoylation and Recycling in Neurodegeneration with Brain Iron Accumulation. Am J Hum Genet 2018, 102(2):266-277.

10. Efferth T, Dunstan H, Sauerbrey A, Miyachi H, Chitambar CR: The anti-malarial artesunate is also active against cancer. Int J Oncol 2001, 18(4):767-773.

11. Sordi R, Nandra KK, Chiazza F, Johnson FL, Cabrera CP, Torrance HD, Yamada N, Patel NS, Barnes MR, Brohi K et al: Artesunate Protects Against the Organ Injury and Dysfunction Induced by Severe Hemorrhage and Resuscitation. Ann Surg 2017, 265(2):408-417.

12. Wan RJ, Li YH: Effects of Artesunate prevent nephritis via the Toll-like receptor 4/nuclear factor-kB signaling pathway in rats. Mol Med Rep 2017, 16(5):6389-6395.

13. Kasaragod VB, Hausrat TJ, Schaefer N, Kuhn M, Christensen NR, Tessmer I, Maric HM, Madsen KL, Sotriffer C, Villmann C et al: Elucidating the Molecular Basis for Inhibitory Neurotransmission Regulation by Artemisinins. Neuron 2019, 101(4):673-689.e611.

14. Zuo S, Ge H, Li Q, Zhang X, Hu R, Hu S, Liu X, Zhang JH, Chen Y, Feng H: Artesunate Protected BloodBrain Barrier via Sphingosine 1 Phosphate Receptor 1/Phosphatidylinositol 3 Kinase Pathway After Subarachnoid Hemorrhage in Rats. Mol Neurobiol 2017, 54(2):1213-1228.

15. Zhang K, Yang Y, Ge H, Wang J, Chen X, Lei X, Zhong J, Zhang C, Xian J, Lu Y et al: Artesunate promotes the proliferation of neural stem/progenitor cells and alleviates Ischemia-reperfusion Injury through PI3K/Akt/FOXO-3a/p27(kip1) signaling pathway. Aging (Albany NY) 2020, 12(9):8029-8048.

16. Paik JH, Ding Z, Narurkar R, Ramkissoon S, Muller F, Kamoun WS, Chae SS, Zheng H, Ying H, Mahoney $\mathrm{J}$ et al: FoxOs cooperatively regulate diverse pathways governing neural stem cell homeostasis. Cell Stem Cell 2009, 5(5):540-553. 
17. Zhang W, Bergamaschi D, Jin B, Lu X: Posttranslational modifications of p27kip1 determine its binding specificity to different cyclins and cyclin-dependent kinases in vivo. Blood 2005, 105(9):3691-3698.

18. Tomoda K, Kubota Y, Kato J: Degradation of the cyclin-dependent-kinase inhibitor p27Kip1 is instigated by Jab1. Nature 1999, 398(6723):160-165.

19. Zhong J, Li RW, Wang J, Wang Y, Ge HF, Xian JS, Feng H, Tan L: Neuroprotection by cattle encephalon glycoside and ignotin beyond the time window of thrombolysis in ischemic stroke. Neural Regen Res 2021, 16(2):312-318.

20. Yeo SS, Choi BY, Chang CH, Jung YJ, Ahn SH, Son SM, Byun WM, Jang SH: Periventricular white matter injury by primary intraventricular hemorrhage: a diffusion tensor imaging study. Eur Neurol 2011, 66(4):235-241.

21. Swanson RA, Morton MT, Tsao-Wu G, Savalos RA, Davidson C, Sharp FR: A semiautomated method for measuring brain infarct volume. J Cereb Blood Flow Metab 1990, 10(2):290-293.

22. Liang T, Chen Q, Li Q, Li R, Tang J, Hu R, Zhong J, Ge H, Liu X, Hua F: 5-HT1a activation in PO/AH area induces therapeutic hypothermia in a rat model of intracerebral hemorrhage. Oncotarget 2017, 8(43):73613-73626.

23. Moskowitz MA, Lo EH, ladecola C: The science of stroke: mechanisms in search of treatments. Neuron 2010, 67(2):181-198.

24. Zhang J, Sun X, Wang L, Wong YK, Lee YM, Zhou C, Wu G, Zhao T, Yang L, Lu L et al: Artesunateinduced mitophagy alters cellular redox status. Redox Biol 2018, 19:263-273.

25. Zuo S, Li Q, Liu X, Feng H, Chen Y: The Potential Therapeutic Effects of Artesunate on Stroke and Other Central Nervous System Diseases. Biomed Res Int 2016, 2016:1489050.

26. Eckert A, Huang L, Gonzalez R, Kim HS, Hamblin MH, Lee JP: Bystander Effect Fuels Human Induced Pluripotent Stem Cell-Derived Neural Stem Cells to Quickly Attenuate Early Stage Neurological Deficits After Stroke. Stem Cells Transl Med 2015, 4(7):841-851.

27. Bacigaluppi M, Russo GL, Peruzzotti-Jametti L, Rossi S, Sandrone S, Butti E, De Ceglia R, Bergamaschi A, Motta C, Gallizioli M et al: Neural Stem Cell Transplantation Induces Stroke Recovery by Upregulating Glutamate Transporter GLT-1 in Astrocytes. J Neurosci 2016, 36(41):10529-10544.

28. Chang DJ, Lee N, Park IH, Choi C, Jeon I, Kwon J, Oh SH, Shin DA, Do JT, Lee DR et al: Therapeutic potential of human induced pluripotent stem cells in experimental stroke. Cell Transplant 2013, 22(8):1427-1440.

29. Sakata H, Narasimhan P, Niizuma K, Maier CM, Wakai T, Chan PH: Interleukin 6-preconditioned neural stem cells reduce ischaemic injury in stroke mice. Brain 2012, 135(Pt 11):3298-3310.

30. Fuentealba LC, Obernier K, Alvarez-Buylla A: Adult neural stem cells bridge their niche. Cell Stem Cell 2012, 10(6):698-708.

31. Couillard-Despres S, Winner B, Schaubeck S, Aigner R, Vroemen M, Weidner N, Bogdahn U, Winkler J, Kuhn HG, Aigner L: Doublecortin expression levels in adult brain reflect neurogenesis. Eur $\mathrm{J}$ Neurosci 2005, 21(1):1-14. 
32. Takagi T, Yoshimura S, Sakuma R, Nakano-Doi A, Matsuyama T, Nakagomi T: Novel Regenerative Therapies Based on Regionally Induced Multipotent Stem Cells in Post-Stroke Brains: Their Origin, Characterization, and Perspective. Transl Stroke Res 2017, 8(6):515-528.

33. Ferber EC, Peck B, Delpuech O, Bell GP, East P, Schulze A: FOXO3a regulates reactive oxygen metabolism by inhibiting mitochondrial gene expression. Cell Death Differ 2012, 19(6):968-979.

34. Roy S, Kaur M, Agarwal C, Tecklenburg M, Sclafani RA, Agarwal R: p21 and p27 induction by silibinin is essential for its cell cycle arrest effect in prostate carcinoma cells. Mol Cancer Ther 2007, 6(10):2696-2707.

35. Han J, Zhao J, Jiang J, Ma X, Liu X, Wang C, Jiang S, Wan C: Zinc deficiency impairs the renewal of hippocampal neural stem cells in adult rats: involvement of FoxO3a activation and downstream p27(kip1) expression. J Neurochem 2015, 134(5):879-891.

\section{Figures}


A

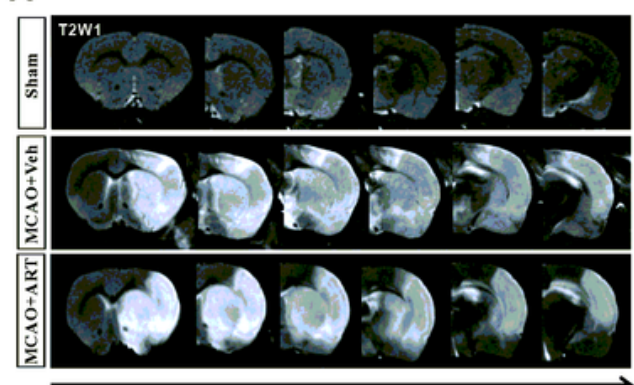

Anterior $2.54 \mathrm{~mm} \quad$ Posterior $-2.46 \mathrm{~mm}$

C

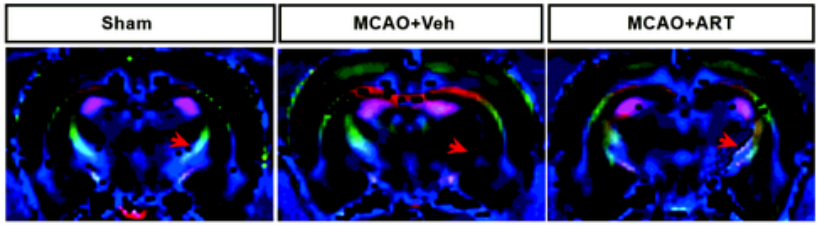

D
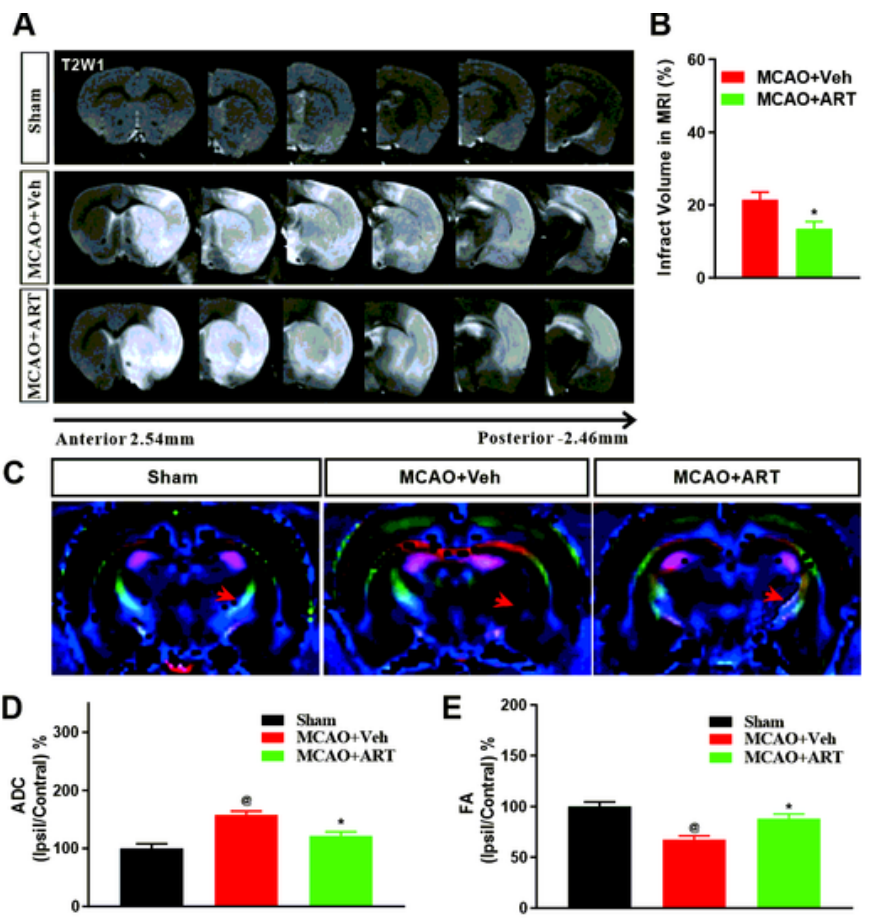

$\mathbf{F}$

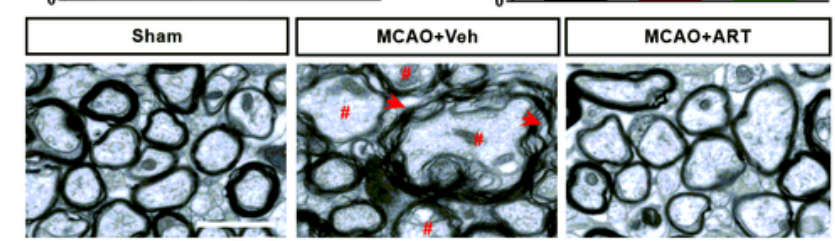

$\mathbf{G}$
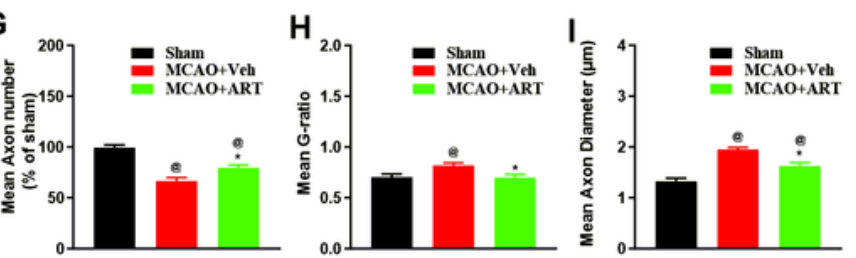

$\sqrt{2}$

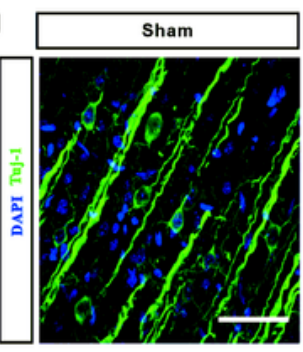

MCAO+Veh
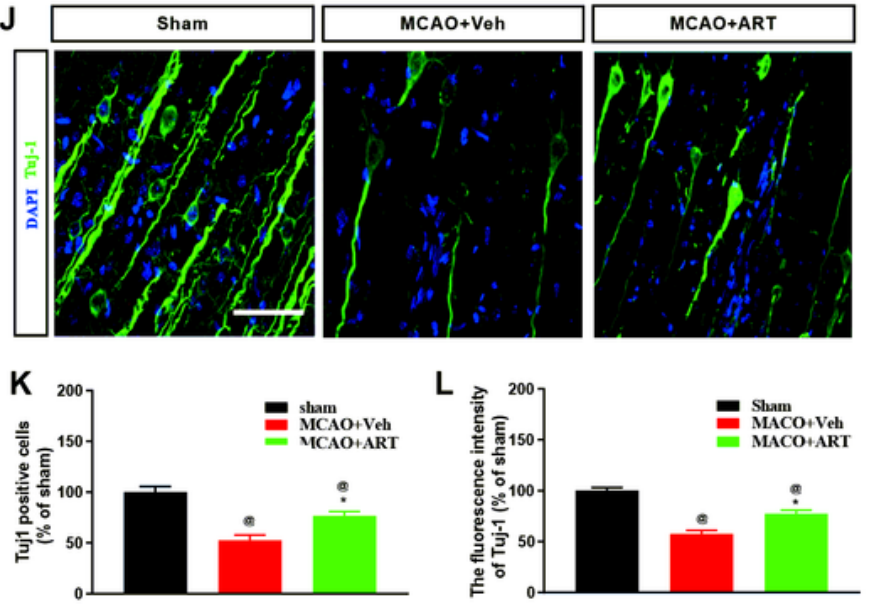

Figure 1

ART treatment reduced cerebral infarct volume and alleviated white matter lesions in the internal capsule of MCAO mice. (A-B) ART treatment remarkably alleviated the ischemic volume by quantifying the cerebral infarct volume in T2-weighed imaging. (C) The nerve fiber bundles were evaluated in the ipsilateral hemisphere using diffusion tensor imaging (DTI) three days following MCAO. The red arrow indicated the injured white matter around the hematoma. (D, E) $150 \mathrm{mg} / \mathrm{kg}$ of ART prevented the reduction 
of apparent diffusion coefficient value (ADC), and fractional anisotropy value (FA) values were reversed by ART treatment. (F) Electron microscopic analysis of white matter axon and myelin sheath at day 3 (scale bar $=2 \mu \mathrm{m}$ ) revealed axons with damaged myelin. The red arrow indicated typically injured myelin and the red \# indicated typically swollen mitochondria. (G-I) Quantitative analysis of mean axonal numbers (per 100 $\mathrm{m} 2$ ), g-ratios, and axonal diameters. The mean axonal number was significantly increased by ART treatment. The mean G-ratio (ratio of axon diameter to total outer diameter); and axon diameter were reduced compared with the MCAO+Veh group. (J) Immunofluorescence staining of Tuj-1. (scale bar=10 $\mu \mathrm{m})$. (K and L) Quantification of DAPI+ cells and fluorescence intensity of Tuj-1 staining. The number of DAPI+ cells and the fluorescence intensity of Tuj-1 were significantly increased in the $M C A O+A R T$ group. Data were presented as mean $\pm S D$ ( $n=6$ in each group). ${ }^{\star} p<0.05$ vs. MCAO+Veh group. $@ p<0.05$ vs. sham group). ART: artesunate. 
A

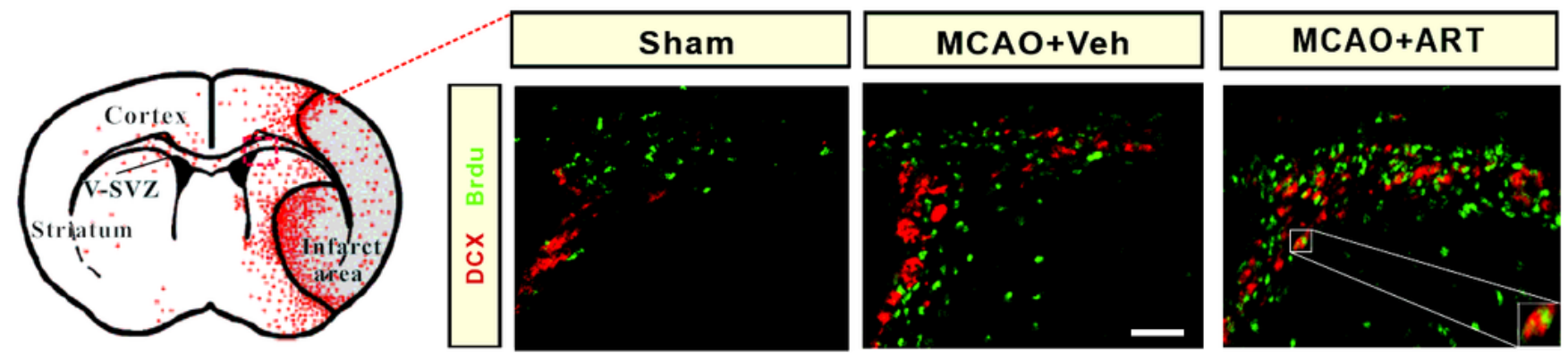

B

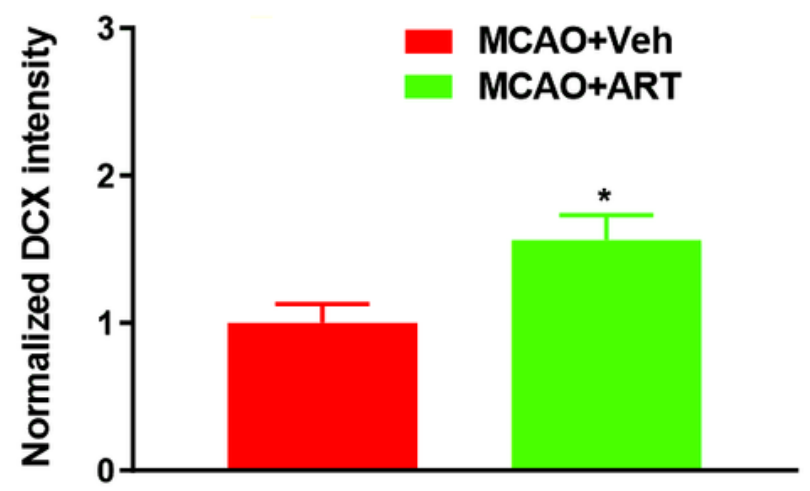

C

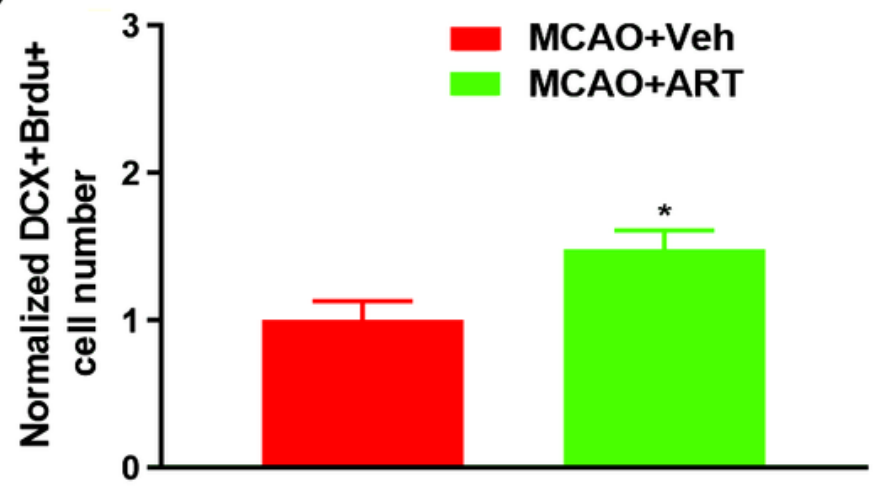

D

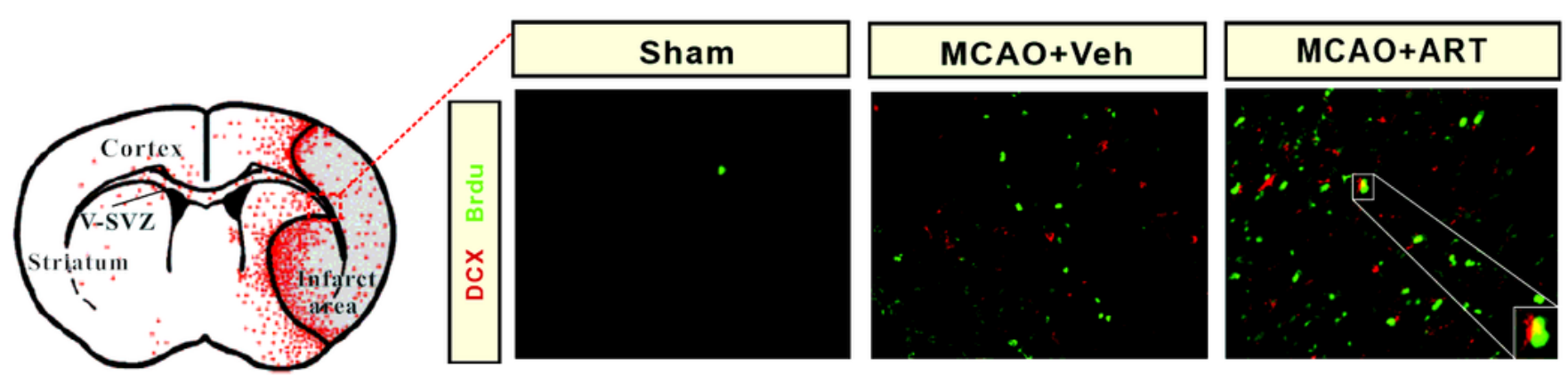

$\mathbf{E}$

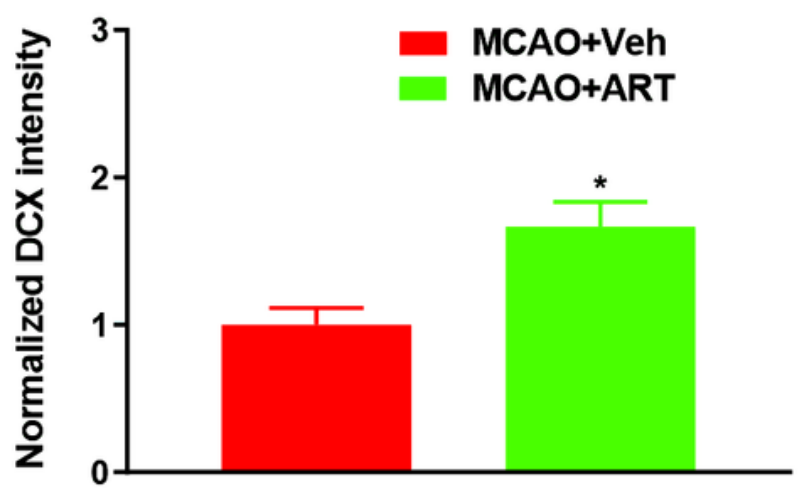

$\mathbf{F}$

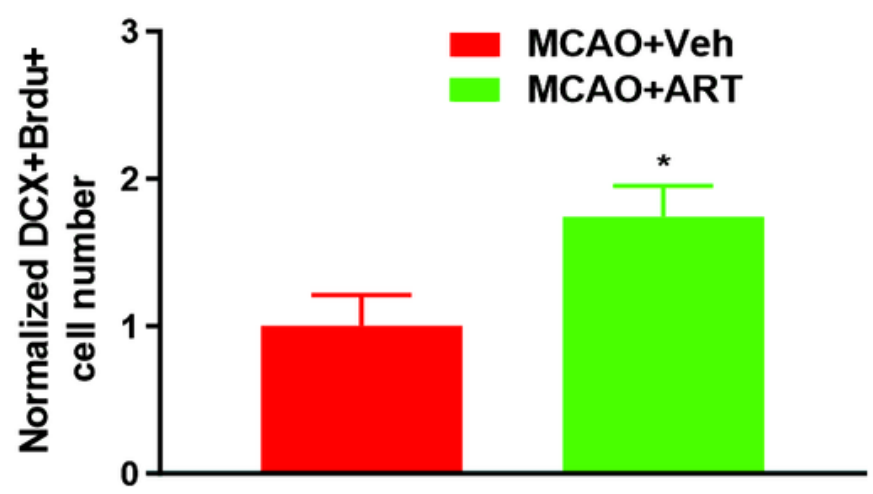

Figure 2

ART treatment promoted neurogenesis in SVZ and the perilesional cortex after MCAO. (A) Representative immunostaining of DCX+ and Brdu+ in the ipsilateral subventricular zone (SVZ) 3 days after MCAO (Scale bar=100 $\mu \mathrm{m}$ ). (B and C) Quantitative analyses of the fluorescence intensity of doublecortin (DCX) and the numbers of DCX+ and Brdu+ cells and ART treatment significantly increased the normalized DCX intensity and the numbers of DCX+ and Brdu+ cells in ipsilateral SVZ (D). Representative immunostaining 
of $\mathrm{DCX}+$ and Brdu+ in perilesional cortex three days post-MCAO (Scale bar $=150 \mu \mathrm{m})$. (E and F) Quantification of the fluorescence intensity of DCX and the numbers of DCX+ and Brdu+ cells and MCAO+ART group showed notably increased numbers of DCX + and Brdu+cells. They elevated DCX intensity in the peri-lesioned cortex on day 3 after MCAO. $n=6$ in each group, ${ }^{*} p<0.05$ vs. MCAO+Veh group. ART: artesunate.

A

FOXO3a

p27 $7^{\text {kip1 }}$

Cyclin E

CDK2

Nestin

GAPDH

ART $-\quad++$

$\mathrm{FOXO3a}^{\mathrm{OE}}---+$

B

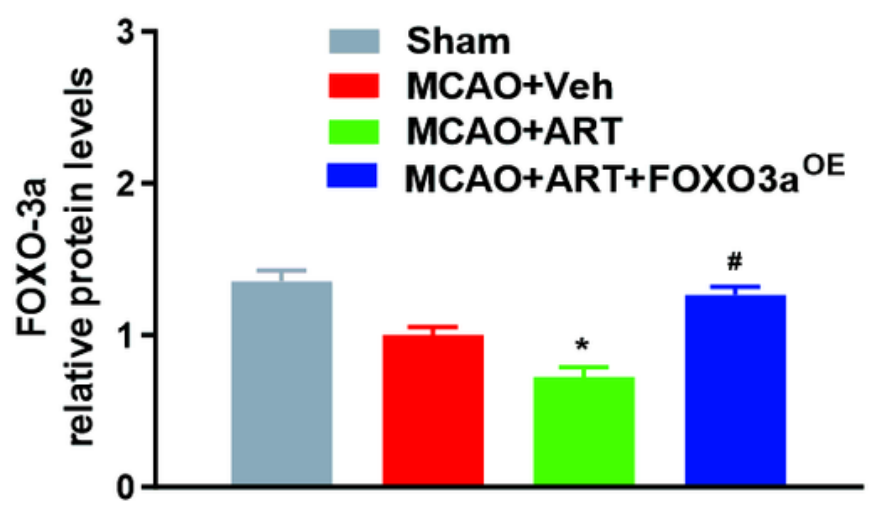

C
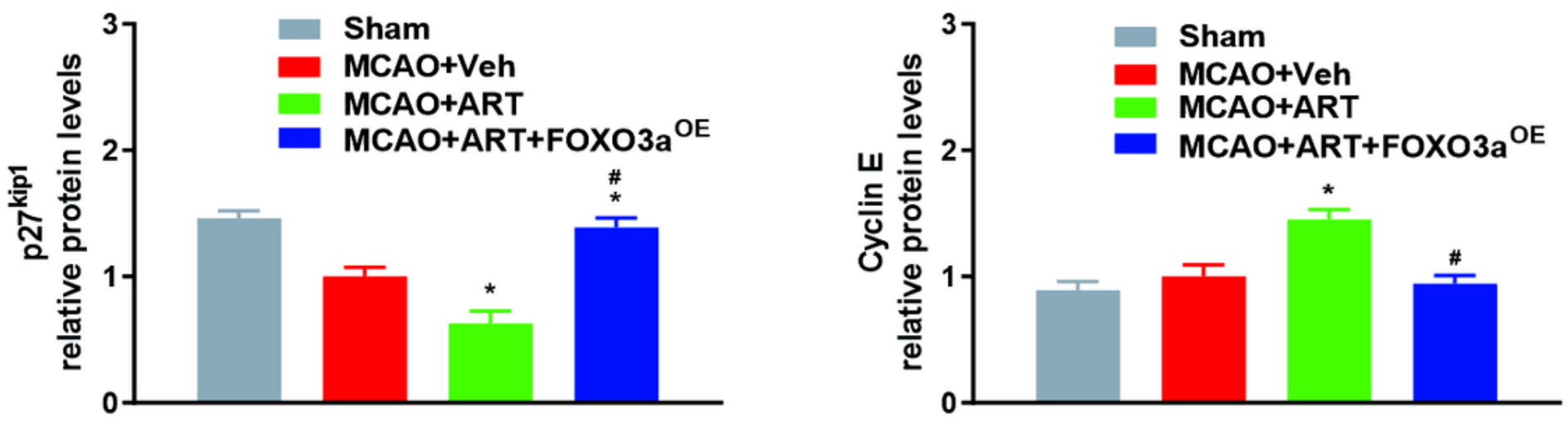

E

$\mathbf{F}$

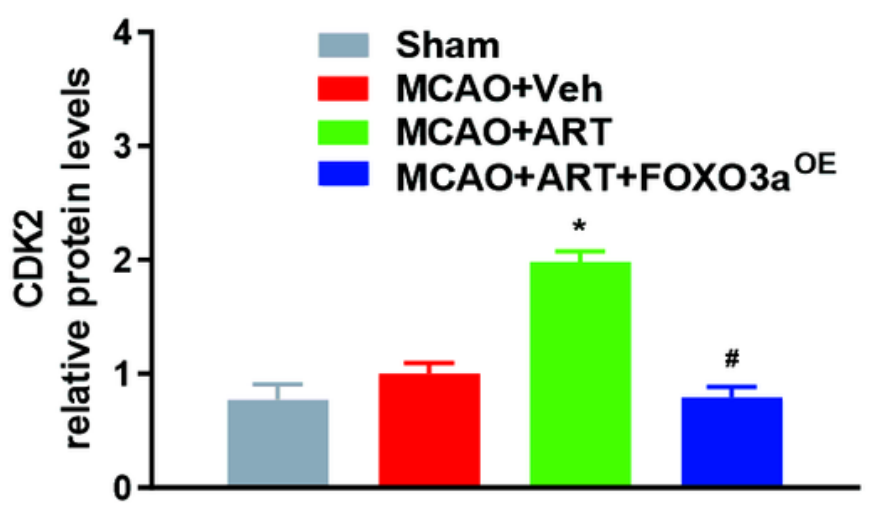

Figure 3 
Expression levels of FOXO3a/p27kip1/Cyclin E/CDK2-associated molecules and Nestin. (A) Representative western blotting for related proteins in Sham, MCAO+Veh, MCAO+ART, and MCAO+ART+ FOXO3aOE group in infarcted cortex three days after surgery. (B-G) Quantitative analysis of western blots: $B$ for FOXO3a, C for p27kip1, D for Cyclin E, E for CDK2, F for Nestin). $n=6$ in each group. ${ }^{*} p<0.05$ vs. MCAO+Veh group; \#p<0.05 vs. MCAO+ART group. ART: artesunate; FOXO3aOE: overexpression model of FOXO3a generated using mutant type Ad-TM-FOXO3a recombinant adenovirus vectors.

\section{A}
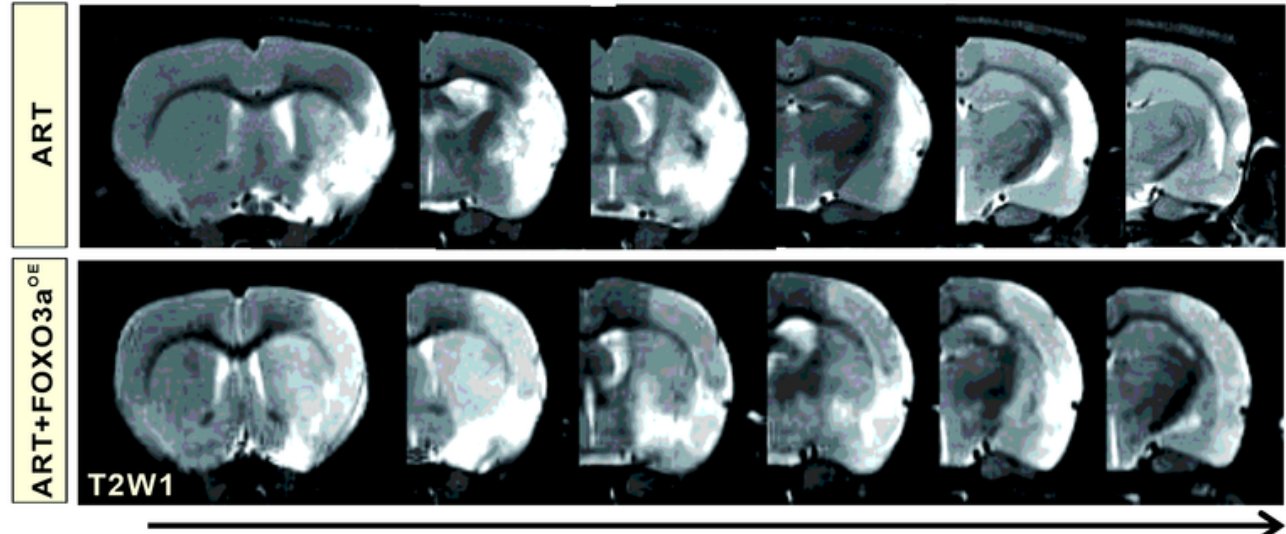

Anterior $\mathbf{2 . 5 4} \mathrm{mm}$

Posterior $\mathbf{- 2 . 4 6 \mathrm { mm }}$
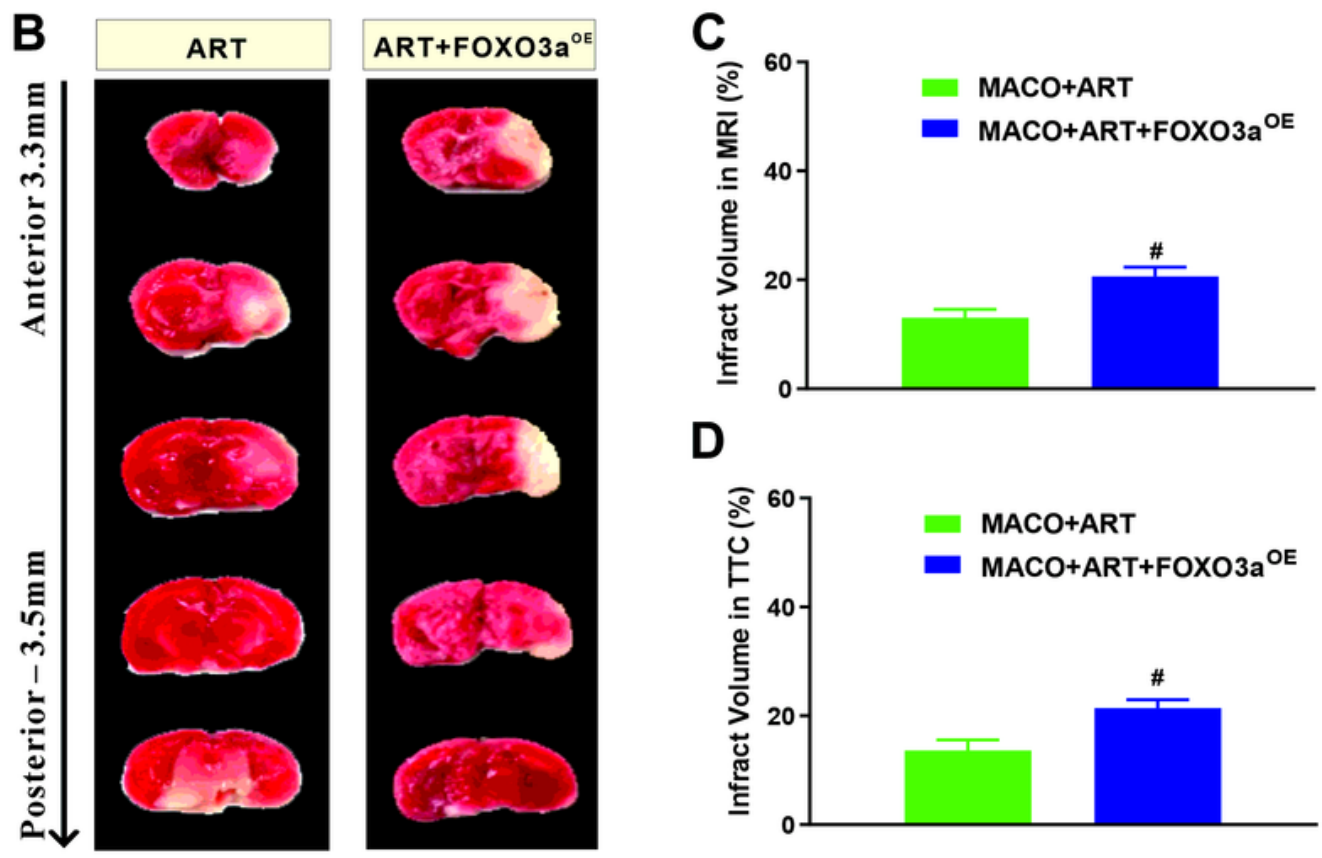

D
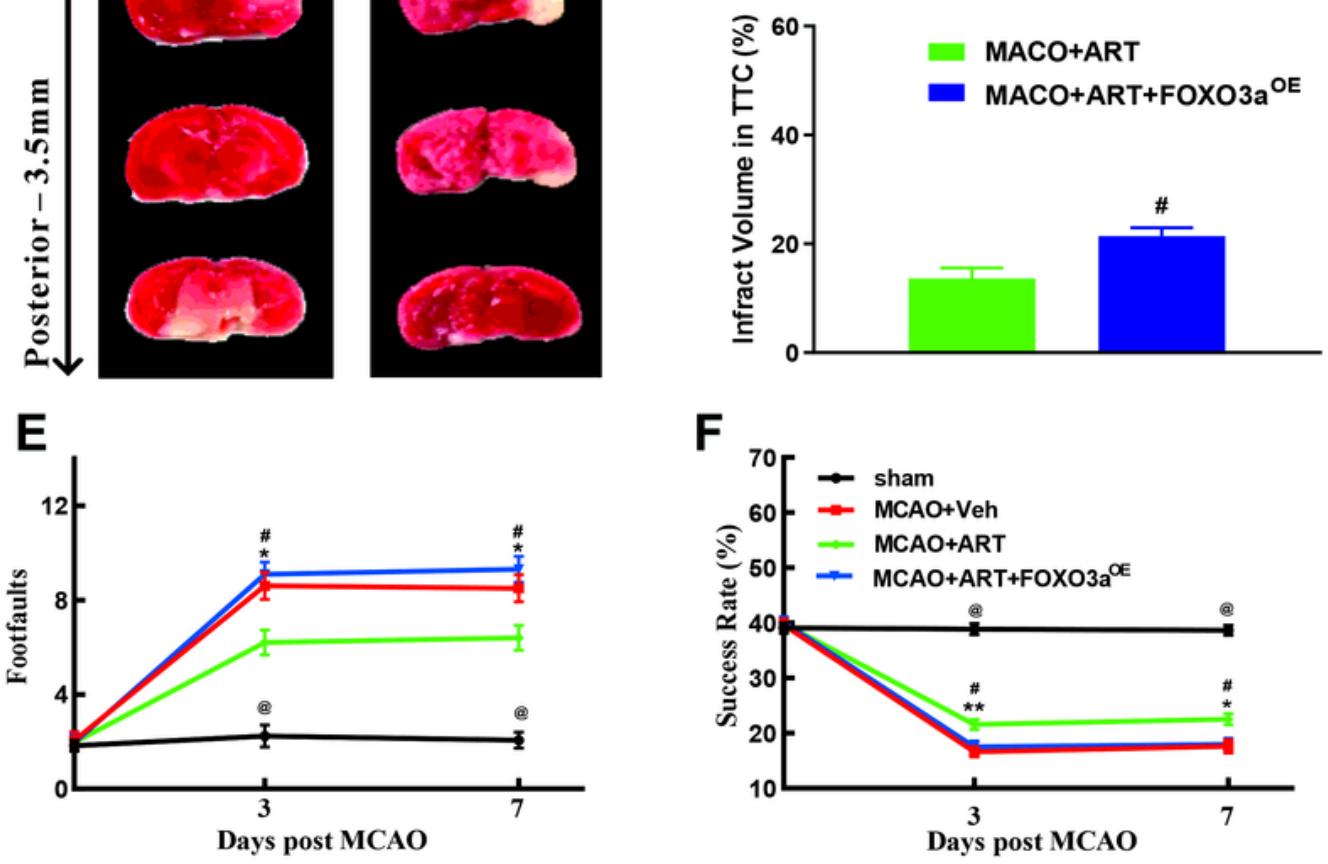

Figure 4 
FOXO3a was involved in ART-modulated cerebral infarct volume change and motor dysfunction. (A and B) The cerebral infarct volume was visualized by T2-weighed imaging and TTC staining three days after MCAO. (C and D) Quantification of cerebral infarct volume. Overexpression of FOXO3a reversed ARTinduced reduction of cerebral infarct volume. $n=6$ in each group. ( $E$ and $F$ ) The grid-walking task and single-pellet retrieval task were performed to examine fine motor function. $n=6$ in each group. $\# p<0.05$ vs. MCAO+ART group. ART: artesunate; FOXO3aOE: overexpression model of FOXO3a generated using mutant type Ad-TM-FOXO3a recombinant adenovirus vectors.

\section{A}

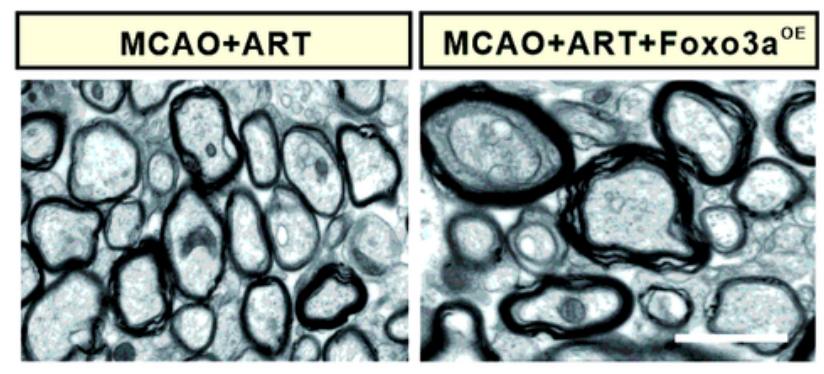

B

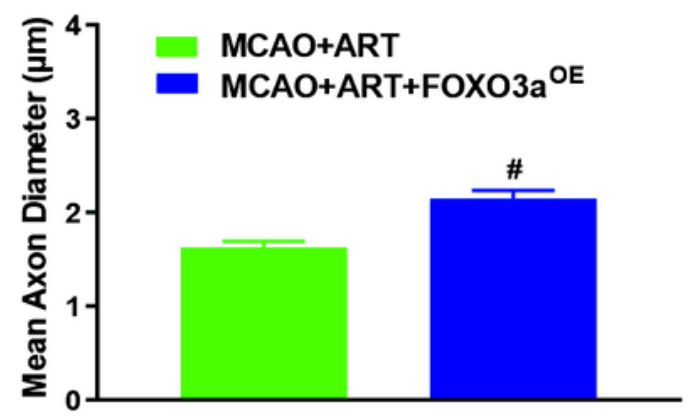

C
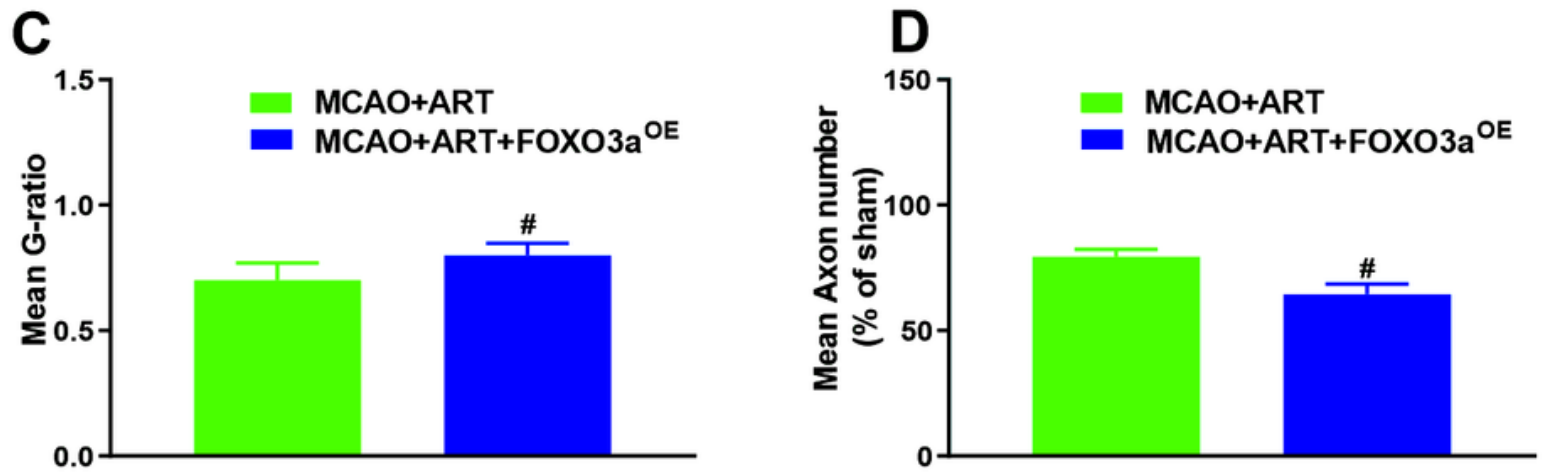

E
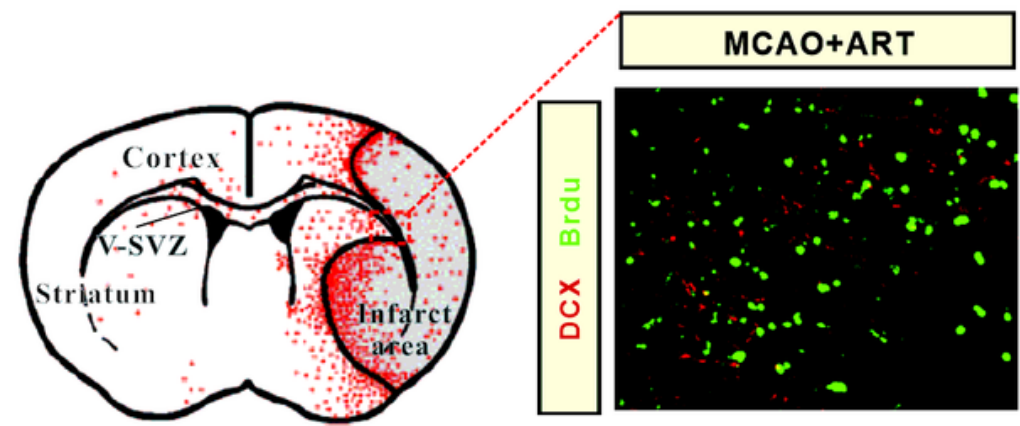

MCAO+ART+FoxO3a ${ }^{O E}$

F
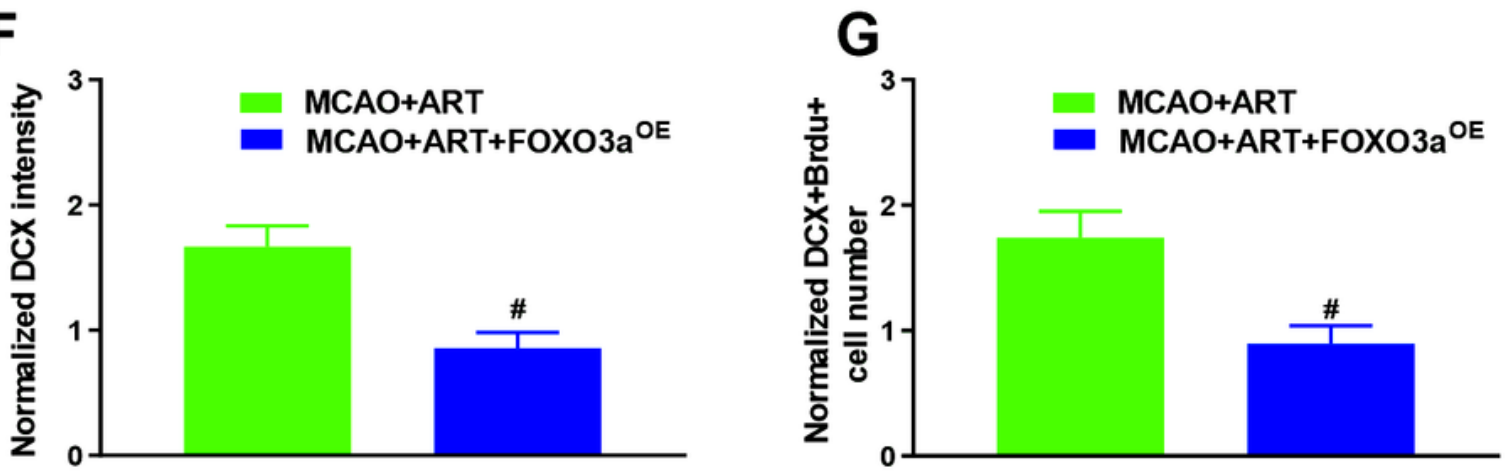


\section{Figure 5}

FOXO3a was involved in ART-regulated white matter lesions and neurogenesis. (A) Electron microscopic analysis of white matter axon and myelin sheath three days after MCAO (scale bar=2 $\mu \mathrm{m})$. (B-D)

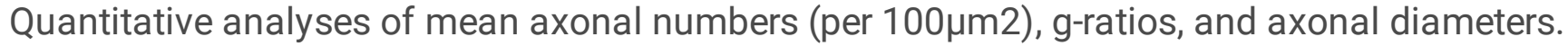
Overexpressed FOXO3a significantly reduced ART's protective effects on axon swelling and myelin injury in white matter fiber, and the numbers of the mean axon, G-ratio, and axon diameter remarkably reversed.

(E) Representative immunostaining of DCX in perilesional cortex three days post-MCAO (scale bar $=50$ $\mu \mathrm{m})$. ( $F$ and $G$ ) Quantitation of normalized DCX intensity and numbers of $D C X+$ and Brdu+ cells. $n=6$ in each group. \#p<0.05 vs. MCAO+ART group. ART: artesunate; FOXO3aOE: overexpression model of FOXO3a generated using mutant type Ad-TM-FOXO3a recombinant adenovirus vectors.
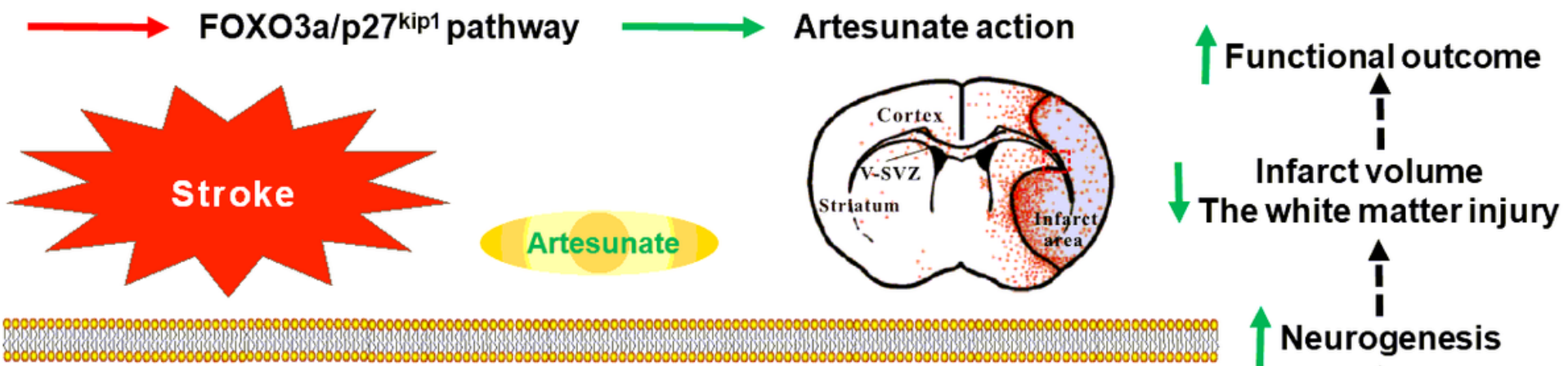

Neurogenesis

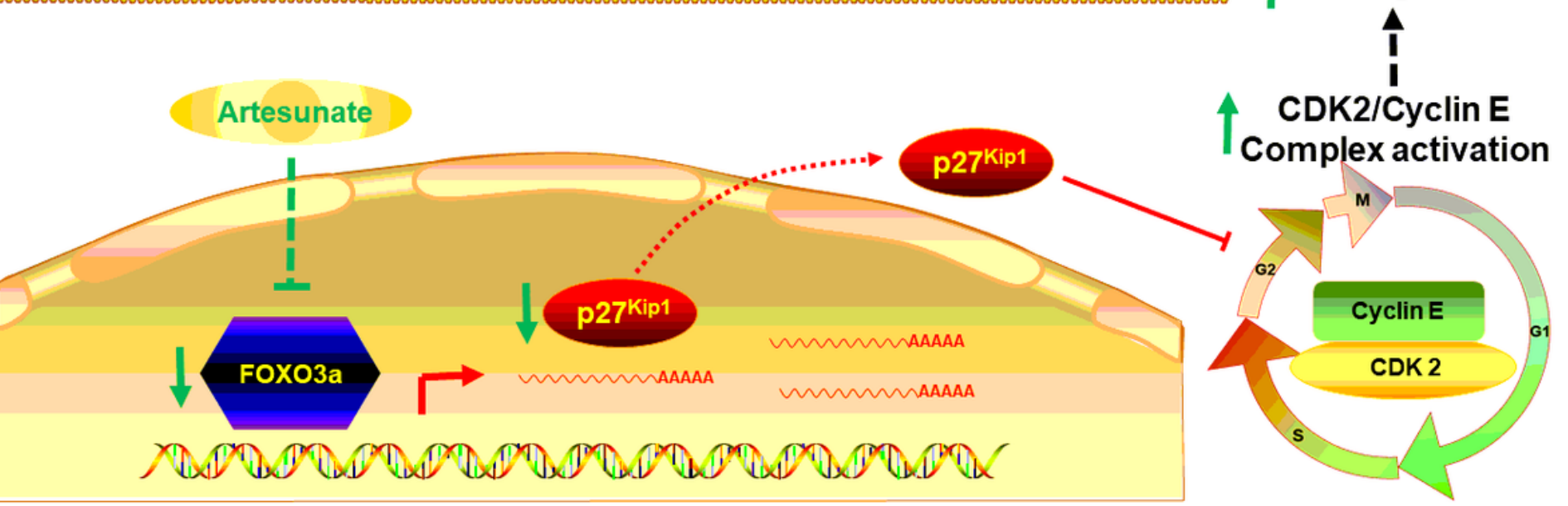

Figure 6

Schematic summary. After an ischemia/reperfusion injury, the artesunate promoted neurogenesis and proliferation of endogenies NSPCs through FOX03a/p27Kip1 signaling, alleviated the white matter lesions in the infracted internal capsule, reduced infarct volume, and promoted functional reconstruction.

\section{Supplementary Files}

This is a list of supplementary files associated with this preprint. Click to download.

- supplymental.pdf 